Supporting information for:

\title{
Probing the radial chemistry of getter components in light water reactors via controlled electrochemical dissolution
}

Sayandev Chatterjee, ${ }^{a}$ Meghan S Fujimoto, ${ }^{a, c}$ Nathan L Canfield, ${ }^{a}$ Monte $R$ Elmore, ${ }^{a}$ Tamas Varga, ${ }^{b}$ Gary J Sevigny, ${ }^{a}$ David J Senor ${ }^{a}$

${ }^{a}$ Energy and Environment Directorate, Pacific Northwest National Laboratory, Richland, Washington 99352, USA. E-mail: Sayandev.Chatterjee@pnnl.gov

${ }^{\mathrm{b}}$ Environmental Molecular Sciences Laboratory, Pacific Northwest National Laboratory, Richland, Washington 99352, USA

'Present address: Idaho National Laboratory, 1955 N Fremont Avenue, Idaho Falls ID 83415, USA.
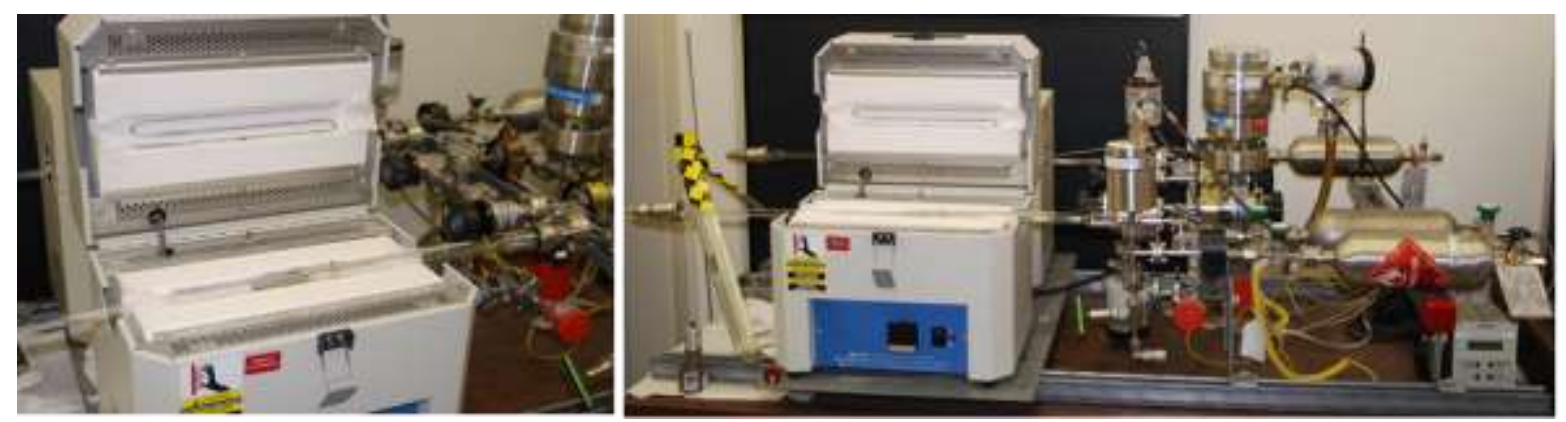

Figure S1. Instrumentation of the tube furnace assembly for loading the liner with specified amounts of H/D.

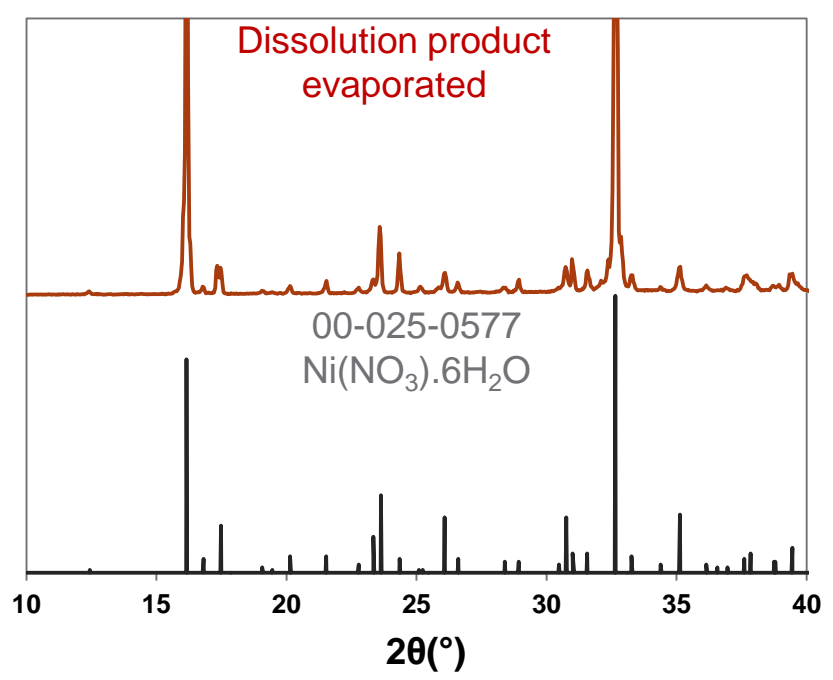

Figure S2. Comparison of experimental XRD patterns with those obtained from literature: (top, brown

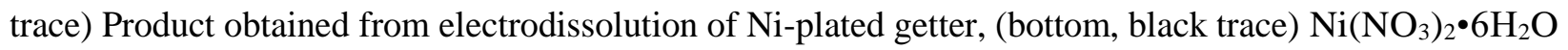
from literature (\#00-025-0577). ${ }^{1}$ 


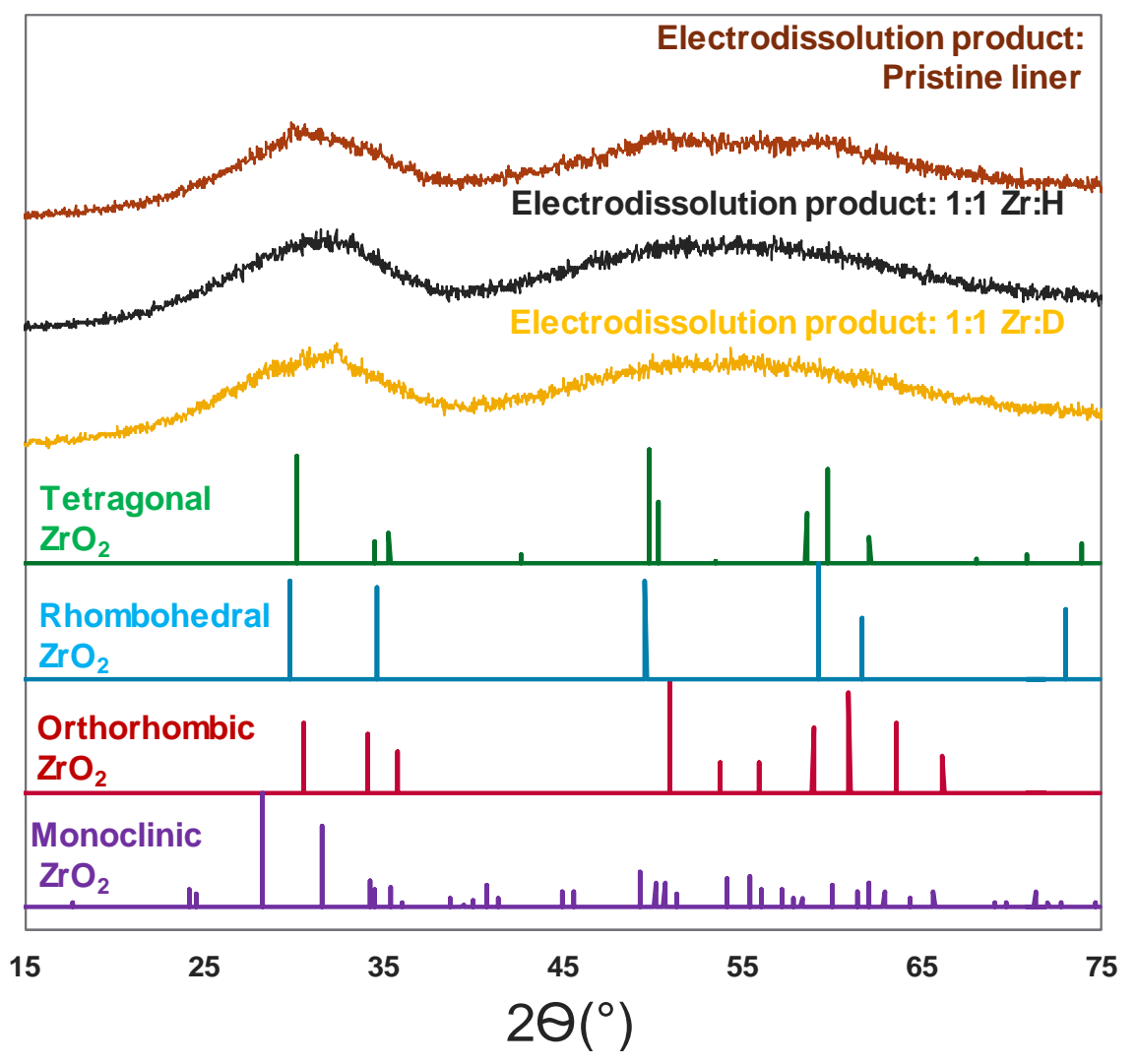

Figure S3. Comparison of experimental XRD patterns with those obtained from literature ${ }^{1}$ : (top, brown trace) Product obtained from electrodissolution of $\mathrm{Zr}-4$ getter, (second from the top, black trace) Product obtained from electrodissolution of H-loaded Zr-4 getter (1: $1 \mathrm{~mol} \mathrm{Zr:H),} \mathrm{(third} \mathrm{from} \mathrm{the} \mathrm{top,} \mathrm{yellow} \mathrm{trace)}$ Product obtained from electrodissolution of D-loaded Zr-4 getter (1: $1 \mathrm{~mol} \mathrm{Zr:D),} \mathrm{(fourth} \mathrm{from} \mathrm{the} \mathrm{top,}$ green trace) tetragonal $\mathrm{ZrO}_{2}$ from literature (\#00-017-0923), ${ }^{2-3}$ (third from the bottom, blue trace) rhombohedral $\mathrm{ZrO}_{2}$ from literature (\#00-037-0031), ${ }^{4}$ (second from the bottom, red trace) orthorhombic $\mathrm{ZrO}_{2}$ from literature (\#00-034-1084), ${ }^{5}$ (bottom, violet trace) monoclinic $\mathrm{ZrO}_{2}$ from literature (\#00-0130307). ${ }^{6}$
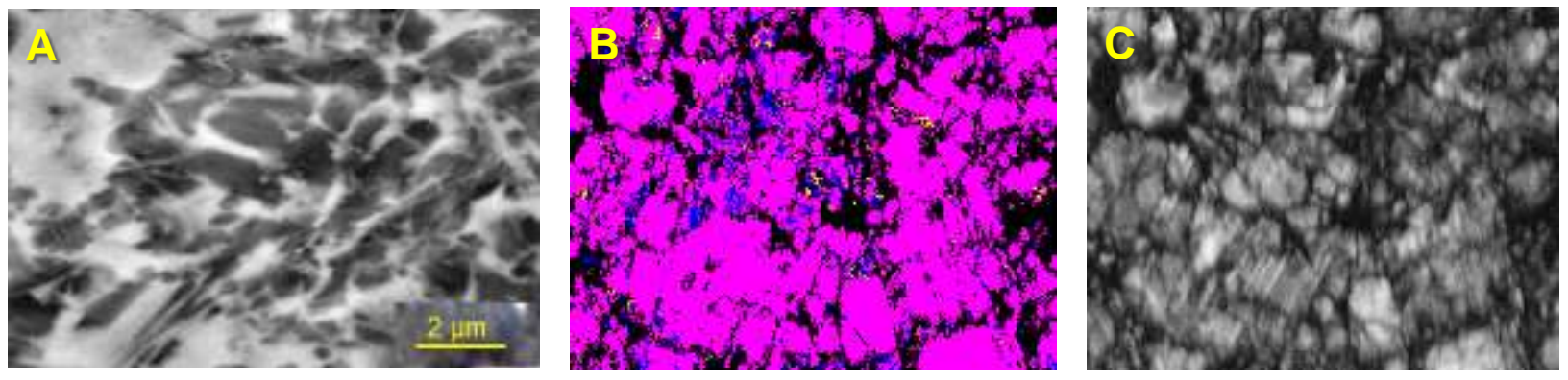

Figure S4. (A) Representative EBSD image of the H-loaded 1:1 mol $\mathrm{Zr:H}$ getter after 12\% electrodissolution in $1 \mathrm{M} \mathrm{KCl}$ using an electrodissolution potential of $275 \mathrm{mV}$ vs. $\mathrm{Ag} / \mathrm{AgCl}$, (B) the phase map of the area represented in image A (the phase distributions shown in Table 4), (C) the pattern-quality map of the aforementioned region. 
Table S1. The quantitative distribution of phases observed in Figure S4 after 12\% dissolution by weight of the H-loaded 1:1 mol Zr:H getter in $1 \mathrm{M} \mathrm{KCl}$ using an electrodissolution potential of $275 \mathrm{mV}$ vs. $\mathrm{Ag} / \mathrm{AgCl}$

\begin{tabular}{|c|c|c|c|}
\hline $\begin{array}{c}\text { Phase } \\
\text { Color }\end{array}$ & Phase & $\begin{array}{c}\text { Raw Area } \\
\%\end{array}$ & $\begin{array}{c}\text { Normalized } \\
\text { Area\% }\end{array}$ \\
\hline & Zirconium - alpha & 3.8 & 5.54 \\
\hline $\begin{array}{c}\text { Zirconium Hydride } \\
(1 / 2) \text { - Epsilon }\end{array}$ & 6.8 & 8.03 \\
\hline $\begin{array}{c}\text { Zirconium Hydride } \\
(1 / 1) \text { - Gamma }\end{array}$ & 9.4 & 11.11 \\
\hline $\begin{array}{c}\text { Zirconium Hydride } \\
(1 / 2) \text { - Delta }\end{array}$ & 64.6 & 76.33 \\
\hline Zero Solutions & 15.3 & - \\
\hline
\end{tabular}




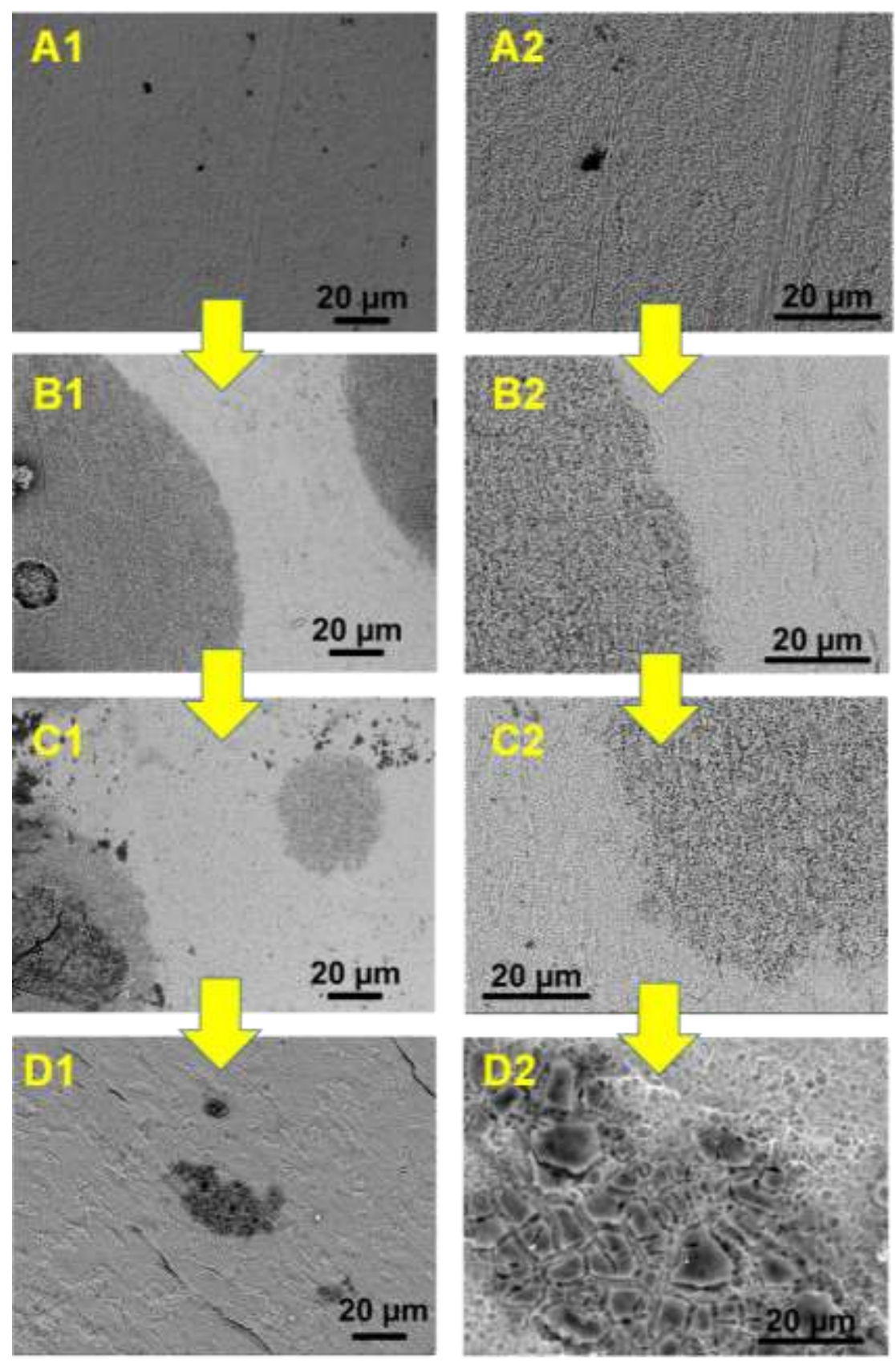

Figure S5. Representative secondary electron SEM images of H-loaded liners 1:1 mol Zr:H at four different stages of dissolution examined at two magnifications: (A1-D1) 150x magnification, (A2-D2) 450x magnification. (A) pristine getter, (B) 12\% dissolution, (C) 35\% dissolution, (D) 63\% dissolution. 


\section{References.}

1. Kabekkodu, S., Powder Diffraction File Inorganic and Organic Data Book. International Center for Diffraction Data: Newtown Square, PA, USA, 2015; Vol. 60.

2. Howard, C. J.; Hill, R. J.; Reichert, B. E., Structures of the $\mathrm{ZrO}_{2}$ Polymorphs at Room-Temperature by High-Resolution Neutron Powder Diffraction. Acta Crystallogr B 1988, 44, 116-120.

3. Teufer, G., Crystal Structure of Tetragonal $\mathrm{ZrO}_{2}$. Acta Crystallogr 1962, 15 (Nov), 1187-1187.

4. Hasegawa, H., Rhombohedral Phase Produced in Abraded Surfaces of Partially Stabilized Zirconia (Psz). J Mater Sci Lett 1983, 2(3), 91-93.

5. Lityagina, L. M.; Kabalkina, S. S.; Pashkina, T. A.; Khozyainov, A. I., Polymorphism of $\mathrm{ZrO}_{2}$ at HighPressure. Fiz Tverd Tela+ 1978, 20(11), 3475-3477.

6. Garvie, R. C., Phase Analysis in Zirconia Systems. J Am Ceram Soc 1972, 55(6), 303-305. 